\title{
Night shift work and breast cancer: a pooled analysis of population-based case- control studies with complete work history
}

(Final version published in Eur J Epidemiol 2018 Apr;33(4):369-379. doi: 10.1007/s 10654018-0368-x)

Emilie Cordina-Duverger, Florence Menegaux, Alexandru Popa, Sylvia Rabstein, Volker Harth, Beate Pesch, Thomas Brüning, Lin Fritschi, Deborah C Glass, Jane S Heyworth, Thomas C Erren, Gemma Castaño-Vinyals, Kyriaki Papantoniou, Ana Espinosa, Manolis Kogevinas, Anne Grundy, John J Spinelli, Kristan J Aronson, Pascal Guénel

\section{Affiliations:}

ECD, FM, AP, PG: Université Paris-Saclay, Université Paris-Sud, CESP (Center for Research in Epidemiology and Population Health), Inserm, Team Cancer-Environment, Villejuif, France

SR, BP, TB: Institute for Prevention and Occupational Medicine of the German Social Accident Insurance, Institute of the Ruhr-Universität Bochum (IPA), Germany.

VH: Institute for Occupational and Maritime Medicine (ZfAM),), University Medical Center Hamburg-Eppendorf (UKE);

LF: School of Public Health, Curtin University, Perth, Australia

DCG: Department of Epidemiology and Preventive Medicine, School of Public Health and Preventive Medicine. Monash University, Melbourne, Australia

JSH: School of Population and Global Health, The University of Western Australia, Crawley, Western Australia

TCE: Institute and Policlinic for Occupational Medicine, Environmental Medicine and Prevention Research, University Hospital of Cologne, University of Cologne, Cologne, Germany

GCV, AE, MK: IS Global, Centre for Research in Environmental Epidemiology (CREAL), Barcelona, Spain; IMIM (Hospital del Mar Medical Research Institute), Barcelona, Spain ; Universitat Pompeu Fabra (UPF), Barcelona, Spain ; CIBER Epidemiología y Salud Pública (CIBERESP), Madrid, Spain

KP: Department of Epidemiology, Medical University of Vienna, Vienna, Austria ; ISGlobal, Centre for Research in Environmental Epidemiology (CREAL), Barcelona, Spain ; Universitat Pompeu Fabra (UPF), Barcelona, Spain ; CIBER Epidemiología y Salud Pública (CIBERESP), Madrid, Spain

AG: CRCHUM (Centre de recherche du CHUM), Montreal, Quebec, Canada; and Department of Social and Preventive Medicine, Université de Montréal, Montreal, Quebec, Canada

JJS: Population Oncology, BC Cancer, Vancouver, British Columbia, Canada ; School of Population and Public Health, University of British Columbia, Vancouver, British Columbia, Canada.

KJA: Department of Public Health Sciences and Cancer Research Institute, Queen's University, Kingston, Ontario, Canada 


\begin{abstract}
Night shift work has been suspected to increase breast cancer risk but epidemiological studies have been inconsistent due to heterogeneous assessment of exposure to night work. To overcome this limitation, we pooled data of five population-based case-control studies from Australia, Canada, France, Germany, and Spain into a single harmonized dataset using a common definition of night work including 6093 breast cancer cases and 6933 population controls.

The odds ratio for breast cancer in women who ever worked at night for at least three hours between midnight and 5 a.m. as compared to never night workers was 1.12 (95\% CI 1.00-1.25). Among pre-menopausal women, this odds ratio was 1.26 [1.06-1.51], increasing to 1.36 [1.07-1.74] for night shifts $\geq 10$ hours, 1.80 [1.20-2.71] for work $\geq 3$ nights/week, and 2.55 [1.03-6.30] for both duration of night work $\geq 10$ years and exposure intensity $\geq 3$ nights/week. Breast cancer risk in pre-menopausal women was higher in current or recent night workers (OR=1.41 [1.06-1.88]) than in those who had stopped night work more than 2 years ago. Breast cancer in post-menopausal women was not associated with night work whatever the exposure metric. The increase in risk was restricted to ER+ tumors, particularly those who were both ER+ and HER2 +.

These results support the hypothesis that night shift work increases the risk of breast cancer in pre-menopausal women, particularly those with high intensity and long duration of exposure. Risk difference between pre- and post-menopausal women deserves further scrutiny.
\end{abstract}

Key words: Night shift work, Breast Cancer, pooled analysis, case-control study, circadian disruption 


\section{Introduction}

Shift work involving circadian disruption was classified as a probable carcinogen (group 2A) by IARC in 2007 based on sufficient evidence in experimental animals and limited evidence in humans [1]. The animal studies showed that variations in circadian rhythm induced by various methods of manipulating the light/dark cycle had an impact on tumor development, and several biological mechanisms could explain how the 'disruption of circadian rhythm' may promote carcinogenesis [2]. The epidemiological studies evaluated in the IARC report mainly addressed breast cancer risk, and found only modest increases in risk, in particular in nurses who had worked at night for 20 years or more [3].

Since the publication of this report, results of additional epidemiological studies on breast cancer in relation to night shift work have been inconsistent [4-17]. Differing characterizations of exposure to night work have been used in these studies in terms of intensity, length of night shifts, number of consecutive nights, and rotation patterns, and not all studies have assessed exposure over the full occupational history (e.g.[13, 15, 18]).

In addition, cohort studies conducted in occupational groups with a specific work time pattern (e.g. nurses) limit extrapolation to other groups with different schedules. Metaanalyses of case-control and cohort studies have reported increases of about $20 \%$ in summary relative risks of breast cancer in women who worked at night [19-22], while a recent meta-analysis of prospective cohort studies did not find any increased risk of breast cancer in women who ever worked at night, or in those who had long duration of exposure to night work [17]. These meta-analyses aggregated findings from studies that used heterogeneous definitions of night work and different exposure metrics, making their combined interpretation difficult.

In the present paper, we conducted a pooled analysis of individual level, rather than aggregated, data from studies of night work and breast cancer by recoding night work to allow a common characterization of exposure across studies. To this end, we followed, the recommendations made by an IARC expert panel [23] for characterizing exposure to night work that impacts on the circadian rhythm. We also focused on general population studies with complete work history to investigate the role of temporal pattern of exposure. 


\section{Material and methods}

\section{Study selection}

We identified 30 publications on night work and breast cancer from a literature search in mid-2016 and excluded six meta-analysis articles that did not provide original data. From the remaining 24 original epidemiological studies, only those with detailed information on work history and work time schedules were considered for inclusion in the pooled analysis. We also sought to include general population studies not restricted to a single occupation to capture a wide range of work time schedules.

We finally retained five recent population-based case-control studies from Australia (BCEES) [9], Canada (CBCS) [7], France (CECILE) [8], Germany (GENICA) [4], and Spain (MCC-Spain) [11] (Table 1). Study investigators provided the original de-identified data for the study participants. The coordinating center was responsible for constituting and maintaining the pooled dataset, i.e. performing quality controls, recoding work time schedules using a common definition of night work, harmonizing data on breast cancer risk factors, and conducting the statistical analyses.

\section{Selection of study subjects and methods of data collection in the original studies}

Details on study design and night work exposure assessment in each study are given in the original papers $[8,7,4,11,9]$. Briefly, the cases were histologically confirmed breast cancer patients recruited from major hospitals in the study areas (France, Canada, Germany, Spain) or from the regional cancer registry (Canada, Australia). The periods of cancer incidence ranged from 2000-2004 at the earliest in Germany to 2008-2013 at the most recent in Spain.

Controls were women with no previous history of breast cancer and were frequencymatched by age to the cases. They were recruited from the same source population as the cases using the telephone directory (France), the electoral roll (Australia), rosters of general practitioners (Spain), population registry (Germany), or among women attending the regional population screening mammography program (Canada). In total, 6093 breast cancer cases and 6933 controls were included in the pooled analysis, representing a total of more than 54,000 job periods. The definitions of night work in the original publications 
differed widely between studies, as can be seen in Table 1. According to these definitions, the proportion of night workers ranged from $7.0 \%$ in Germany to $34.4 \%$ in Canada.

\section{Assessment of exposure to night work in each study and in the pooled analysis}

Information on work time schedules in the original datasets was obtained for each job held for more than six months (twelve months in Spain) in a woman's work history. These questionnaires were administered during in-person interviews in France and Spain. In Canada the questionnaire was either self-administered or administered by phone, depending on the subject preference. In Australia and Germany, women were first asked during an in-person interview (Germany) or self-administered questionnaire (Australia) if they ever worked night shifts, and those women were re-contacted by phone to complete an interview on details of work time schedules.

Data provided by investigators included the year when each job started and ended, the number of working hours per week, and a code for job title. Hours at start and end of work were available for all jobs in the Canadian and Spanish studies, jobs that included at least one working hour between 11 p.m. and 5 a.m. in the French study, jobs that involved "shift work" in the German study, and jobs that included any number of hours between midnight and 5a.m. in the Australian study (Table 1). The number of nights per week in the French, German and Spanish studies was directly obtained from the questionnaire ("how many nights per week or month did you have on average while you were employed in that job?"); in Canada, women were asked to report the "percentage of time worked at each of day, evening or night shifts"; for this study, the number of nights per week was estimated using the formula: (number of work hours per week) x (percent of working time at night) / (length of night shift in hours). No data on the frequency of night shifts was available in the Australian study, which was therefore excluded from the analyses where this variable was used.

In the combined dataset, any job that included three or more working hours between midnight and 5 a.m. was classified as a job involving night work [23]. Based on this definition, we used the following exposure metrics for characterizing a woman's lifetime exposure to night work: duration (i.e. total number of years in jobs involving night work); length of night shifts (mean number of hours between start and end of shift, averaged over the duration of all jobs involving night work); intensity (mean number of night shifts 
per week averaged over the duration of all jobs involving night work (not available for Australia)); and time since last night shift (years between last night shift and date of diagnosis for the cases or date of recruitment for the controls). We also calculated the cumulative number of night shifts during lifetime (intensity x duration) and the average number of night hours per week (intensity x night shift length).

\section{Breast cancer risk factors}

Data on socio-demographic, reproductive, hormonal, anthropometric characteristics, and lifestyle habits associated with breast cancer (age, age at menarche, age at first fullterm pregnancy, parity, duration of breastfeeding, use of menopausal hormone therapy and oral contraceptives, family history of breast cancer in first-degree relatives, alcohol consumption, tobacco smoking, body mass index (BMI) measured from 1 to 2 years before diagnosis depending on the study, and menopausal status) were obtained for each participant. All variables were recoded into identical categories and compiled into a combined dataset.

\section{Statistical Analyses}

Odds ratios and 95\% confidence limits for the association between night work exposure metrics and breast cancer were calculated using conditional logistic regression models stratified by 5-year age group and study. The following categories were used for duration of night work $(<10,10-19, \geq 20$ years $)$, length of night shifts $(<8,8-9, \geq 10$ hours $)$, intensity $(<1,1-2, \geq 3$ nights per week), time since last night shift $(0-2,3-9,10-19, \geq 20$ years), cumulative number of night shifts over the working lifetime $(<300,300-999$, $\geq 1000)$ and average number of night hours per week $(<11,11-19, \geq 20$ hours). Analyses were conducted for all women combined, and for pre- and post-menopausal women separately using women who had never worked at night as the reference exposure group. Women were considered menopausal if at the time of cancer diagnosis (for cases) or study recruitment (for controls) they had not menstruated for twelve or more months, if they had had bilateral oophorectomy, or if they had used menopausal hormone therapy before cessation of menstruation. Analyses were adjusted for age at menarche $(<12,12$, $13, \geq 14)$, age at first full-term pregnancy $(<22,22-24,25-27, \geq 28$ years), parity (nulliparous, 1, 2, 3, $\geq 4$ children), summed duration of breastfeeding (never, $<6$ months, 6-11 months, $\geq 12$ months), use of menopausal hormone therapy (yes, no), use of oral 
contraceptive (yes, no), family history of breast cancer in first-degree relatives (yes, no), current alcohol drinking (yes, no), ever tobacco smoking (yes, no), and body mass index (BMI) $\left(<18.5,18.5-24,25-29, \geq 30 \mathrm{~kg} / \mathrm{m}^{2}\right)$. To test exposure-response trends, we fitted models where duration of night work, frequency of night shift, and length of night shift were introduced as continuous variables in the model, assuming a linear relationship with breast cancer risk. Differences in the effect of shift work by menopausal status were examined by adding the appropriate interaction terms into the model. Heterogeneity between studies was tested using the Cochran's Q test and $I^{2}$ statistic [24].

Analyses were repeated for breast cancer subtypes defined by hormone receptor status (estrogen receptor ER and human epidermal growth factor receptor HER2). Because PR status was not available in all studies, and because most PR-positive tumors were ER-positive, results including PR status were not informative and are not shown.

Sensitivity analyses were also conducted using alternative definitions of night work, i.e. jobs involving one or more working hour or jobs involving five hours (instead of $\geq 3$ hours) between midnight and 5 a.m. All statistical analyses were conducted using SAS software version 9.4 .

\section{Results}

The pooled dataset of all five studies with information on night work included 6093 cases and 6933 controls. The subset of the four studies where the number of nights per week was available (i.e. excluding Australia) comprised 4894 cases and 5154 controls. Selected characteristics of the study populations are shown in supplemental Table S1. Mean age varied between 54.7 years in France to 58.7 years in Spain. The proportion of pre-menopausal women was below 25\% in Australia and Germany, 30\% in Spain and $38 \%$ in France and Canada. The prevalence of obesity (BMI $\geq 30 \mathrm{~kg} / \mathrm{m} 2$ ) ranged from $12 \%$ to $23 \%$. Nulliparous women were $7 \%$ in France and 29\% in Canada. Parity, age at FFTP, breastfeeding, use of oral contraceptives or menopausal hormone therapy differed widely between countries.

Using a common definition of night work across all studies (i.e. classifying as ever night worker any woman with work shifts of at least three hours between midnight and 5 a.m. in any job), the proportion of ever night workers among controls was around $17 \%$ in the Australian and Canadian studies, 9\% in France and Spain, and 6\% in Germany 
(Table 1). These proportions were lower than the values in the original publications due to more stringent definition of night work in this pooled analysis (Table 1).

The pooled odds ratio for breast cancer in women who ever worked at night was 1.12 (95\% CI 1.00-1.25), ranging from 1.00 in Canada and Germany to 1.27 in Australia (Table 2). No heterogeneity between studies was detected ( $\mathrm{Q}$ test $\mathrm{p}=0.44, \mathrm{I}^{2}=0 \%$ ). The pooled OR was 1.26 (95\%CI 1.06-1.51) in pre-menopausal and 1.04 (95\%CI 0.90-1.19) in post-menopausal women ( $\mathrm{p}$ for interaction $=0.16$ ). The ORs were higher in pre- than in post-menopausal women in all studies, with the exception of the German study, which had small numbers. Tests of heterogeneity between studies did not reach statistical significance in either pre- $\left(\mathrm{Q}\right.$ test $\left.\mathrm{p}=0.62, \mathrm{I}^{2}=0 \%\right)$ or post- $\left(\mathrm{Q}\right.$ test $\mathrm{p}=0.21, \mathrm{I}^{2}=31 \%$ ) menopausal women.

The ORs for breast cancer associated with night work exposure metrics are shown in Table 3. In pre- and post-menopausal women combined, breast cancer risk did not increase with lifetime duration of night work (p-trend=0.45) or with length of night shifts (p-trend=0.38). Breast cancer risk in women who were currently working at night or who had recently quit night work ( $\leq 2$ years) (OR=1.26; 95\% CI 1.02-1.55), was higher than that of women who had stopped working at night more than two years ago. Based on the four studies that provided data on night work intensity (i.e. number of nights per week), the OR for breast cancer was 1.26 (95\% CI 0.97-1.63) for women who worked three or more nights per week and 1.28 (95\%CI 1.01-1.60) for women who worked at night for 20 or more hours per week (intensity x length of shifts). No clear increase in risk was seen with lifetime cumulative number of night shifts ( $\mathrm{p}$-trend=0.10).

Table 3 also shows that the associations between night work exposure metrics and breast cancer were restricted to pre-menopausal women. Among these women, elevated odds ratios were seen for length of night shifts $\geq 10$ hours (OR=1.36; 95\%CI 1.07-1.74) and time since last night shift $\leq 2$ years $(\mathrm{OR}=1.41 ; 95 \% \mathrm{CI} 1.06-1.88)$. Restricting the analysis to four studies with data on exposure intensity, elevated odds ratios were apparent for premenopausal women who worked three or more nights per week (OR=1.80; 95\%CI 1.20-2.71), and those who worked 20 or more night hours per week $(\mathrm{OR}=1.57$; 95\%CI 1.11-2.23). All ORs were close to one among post-menopausal women. 
The pooled odds ratios for all exposure metrics based on the four studies with exposure intensity are shown in Supplemental Table S2. In this table, the OR for breast cancer associated with exposure duration $\geq 20$ years reached 1.68 (95\% CI 0.98-2.83), but no major change was observed for length of night shifts and for time since last night shift. The results for night work exposure metrics tabulated for each study separately are shown in Supplemental table S3. No heterogeneity between studies was detected.

We also explored the effect of exposure intensity $\geq 3$ nights per week, stratified on lifetime exposure duration ( $<10$ or $\geq 10$ years), length of night shifts ( $<10$ or $\geq 10$ hours) and time since last night shift ( $\leq 2$ or $>2$ years) based on the four studies with information on exposure intensity (Table 4). Among pre-menopausal women, the ORs associated with $\geq 3$ nights per week were higher in categories with longer exposure duration $(\geq 10$ years: OR=2.55 95\% CI 1.03-6.30 for), longer night shifts ( $\geq 10$ hours/shift: OR=2.15; 95\%CI 1.21-3.84), and in more recent night workers (last night work $\leq 2$ years: $\mathrm{OR}=2.76 ; 95 \% \mathrm{CI}$ 1.38-5.53). High exposure intensity $\geq 3$ nights per week was not associated with postmenopausal breast cancer regardless of duration of exposure to night work, or length of night shift. Of note, the odds ratio associated with high exposure intensity and time since last night work $\leq 2$ years was increased by $58 \%$ in postmenopausal women, although the increase was not statistically significant possibly because of small numbers.

Table 5 shows ORs associated with ever night work for breast cancer subtypes defined by hormone receptor status. Information on the ER status and HER2 status was missing in 76 and 181 cases, respectively. An elevated OR was seen for ER-positive tumors $(\mathrm{OR}=1.19 ; 95 \% \mathrm{CI}$ 1.06-1.33) among all participants, but the elevation in risk was restricted to pre-menopausal women (OR=1.36; 95\%CI 1.12-1.64). When ER-positive tumors were further subdivided into HER2-positive and HER2-negative tumors, elevated ORs were observed for the ER+/HER2+ tumor subtype in both pre- and in postmenopausal women $(\mathrm{OR}=1.77$; 95\%CI 1.16-2.70; and, $\mathrm{OR}=1.59 ; 95 \% \mathrm{CI} 1.11-2.28$, respectively). Exposure to night work was not associated with the ER-negative breast cancer subtype.

Finally, we also performed sensitivity analyses using varying definitions of night work, i.e. $\geq 1$ hour or 5 hours between midnight and 5 a.m., and results changed only slightly (Supplemental Table S4). 


\section{Discussion}

The major strengths of this pooled analysis are its large sample size including a large number of premenopausal women, detailed information of work time schedules over the entire work history, and use of a common definition of night work that led to a harmonized dataset with population-based individual-level information. When compared to never night workers, we found that the incidence of breast cancer for women who ever worked at night increased by $12 \%$. This increase was stronger among premenopausal women, particularly those with $\geq 3$ nights per week intensity of exposure. In contrast, no association between night work and breast cancer was detected among post-menopausal women when tumour type was not considered. The association of night work with breast cancer was restricted to the ER+ tumors subtypes, particularly those that were both ER+ and HER2+, for both pre- and post-menopausal women.

Despite convincing evidence from experimental animal studies supporting a link between circadian disruption and cancer [1], results of epidemiological studies published to date have been inconsistent, with some studies $[6,25,5,13,16,10]$, but not all $[17,14$, 12], reporting an association between night work and breast cancer. Variations in the definitions of night work and variations in exposure assessment methods can be a source of inconsistency between study findings. Some studies used job-exposure matrices to assess exposure to night work based on the women's occupational title and industry [26, $27,12]$ which may lead to misclassification errors; other studies analyzed the duration of night work $[28,3,29,16]$ without consideration of other surrogate measures of circadian disruption such as intensity of night work, length of night shifts, or frequency of rotations in shifts [23]. Moreover, incomplete assessment of exposure in previous studies has prevented the examination of specific exposure windows during a lifetime [17]. In addition to these limitations, the results of several cohort studies that focused on one particular occupational group (e.g. nurses [3, 30], military [6], textile workers [14], and radio and telegraph operators [31]) may not be applicable to other groups with different patterns of work time schedules.

Meta-analyses based on aggregated data of case-control and cohort studies have reported increased meta-RRs of breast cancer ranging from 10-20\% for night work or shift work, depending on the studies included [19-22, 14]. However, heterogeneity between studies was observed, with higher meta-RRs for case-control than for cohort 
studies [19]. In another recent meta-analysis restricted to large prospective cohort studies, and including mainly post-menopausal women, the meta-RR for breast cancer was not increased, and no association with duration of night shift work was observed [17]. Because the definition of night work and the methods of exposure assessment have been different across studies, meta-analyses based on aggregated data are difficult to interpret.

To overcome these limitations, we re-coded exposure to night work of each participant in five epidemiological studies. The definition of at least three working hours between midnight and 5 a.m. recommended by an IARC expert panel [23] was used to calculate several night work exposure metrics. We found that night work characteristics indicative of high exposure intensity ( 3 or more nights per week) and long night shifts (10 or more hours) were associated with an increased risk of breast cancer in premenopausal women. This is a novel finding indicating that an elevated number of nights worked per week and long working hours at night may induce stronger disruption of circadian rhythm [23], one of the mechanisms by which night work may increase the risk of cancer [2]. Our data also suggested that long duration of night work over lifetime ( $\geq 10$ years) combined with high intensity (average of 3 or more nights per week) was associated with increased breast cancer risk in pre-menopausal women. Taken together, our findings were consistent with the Nurses' Health Study II showing that nurses who worked rotating night shifts for at least 3 nights per month for 20 or more years, particularly during young adulthood, were at increased risk of breast cancer [32]. Several studies [28, $3,16,30,25]$ also reported increased risks of breast cancer associated with long duration of exposure.

Our data also suggested a greater increase in risk among women currently or recently ( $\leq 2$ years) working night shifts, than in those who had stopped working night shifts more than 2 years ago. This temporal pattern may explain at least partly the risk difference between pre- and postmenopausal women, as the latters include a large proportion of women who have stopped working at night for a long period of time. We also observed (Table 4) that the few postmenopausal women who did not stop working at night in the last 2 years and who had worked for $\geq 3$ nights/week had an increased risk of breast cancer, although the increase was not statistically significant.

In line with our observation, it has already been suggested that the null results observed in the meta-analysis of prospective cohort studies by Travis et al. [17] may be 
explained by the older age of the women included in these studies, reflecting exposure to night work that occurred a considerable time in the past [33]. Other explanations for discrepant findings between pre- and post-menopausal women include higher exposure intensity in the younger generations of women due to rapid expansion of night work during the last few decades, or perhaps night work exposures in early adulthood impacting risk more due to an increased susceptibility of breast tissue to circadian disruption in earlier life stages. Divergent findings may also be explained by interactions with risk factors for breast cancer that behave differently between pre- and postmenopausal women (e.g. BMI) or with lifestyle or reproductive factors that are distributed differently for younger and older women (e.g. alcohol, smoking, parity).

In the analysis by breast cancer subtype defined by hormone receptor status, we found that the association between pre-menopausal breast cancer and night work was stronger in ER-positive tumors, pointing to a possible role of estrogens in a pathway between night work and breast cancer. Among ER-positive tumors, we also found that night work was more strongly associated with risk of the breast cancer subtype defined by both ER-positive and HER2-positive receptor status, for both pre- and postmenopausal women. The reason for such an association is unclear and requires confirmation, and exploration, by additional studies.

This pooled analysis has several limitations. First, all studies were retrospective in design. However, potential recall bias was minimized by the use of standardized questionnaires to obtain information on working time schedule for each job over a lifetime, without specific focus on night work. Despite the use of a common framework across the five studies for assessing exposure to night work, complete standardization of exposure metrics may not have been achieved because of the different approaches used in each study to obtain information on night work. This may have led to misclassification errors across exposure categories. Finally, we could not investigate all domains that may be important to circadian disruption resulting from night shift work [23], such as information on the type of night work (rotating vs. permanent, speed and direction of rotation), number of consecutive night shifts, or data on sleep habits, exposure to ambient light at night, or chronotype.

Conversely, strengths of this study include the unique dataset compiled from individual data with common assessment of key exposure characteristics, the detailed 
exposure information including shift work intensity and length, the large sample size, particularly of premenopausal women, allowing good statistical power and adjustment for confounders. Sensitivity analyses using various definitions of night work and various cut-offs of quantitative variables were consistent with the main analysis.

Overall, our study captured important characteristics of night work among many different patterns of shiftwork, and analyzed characteristics that can significantly disrupt the circadian clock $[23,34]$. Intensity of night work, measured as the number of nights per week, was most strongly associated with breast cancer risk among younger women. Our data also suggest that length of night shifts and lifetime duration of night work are important parameters of exposure related to risk, that the risk may decrease after cessation of exposure, and that the risk to specific sub-types of breast cancer (ER-positive tumors) may be affected greater.

Conflict of Interest: The authors declare that they have no conflict of interest.

Ethical approval: All procedures performed in studies involving human participants were in accordance with the ethical standards of the institutional and/or national research committee and with the 1964 Helsinki declaration and its later amendments or comparable ethical standards.

For this type of study formal consent is not required. 


\section{References}

1. IARC. MONOGRAPHS ON THE EVALUATION OF CARCINOGENIC RISKS TO HUMANS: Painting, Firefighting, and Shiftwork2010.

2. Fritschi L, Glass DC, Heyworth JS, Aronson K, Girschik J, Boyle T et al. Hypotheses for mechanisms linking shiftwork and cancer. Medical hypotheses. 2011;77(3):430-6.

3. Schernhammer ES, Kroenke CH, Laden F, Hankinson SE. Night work and risk of breast cancer. Epidemiology. 2006;17(1):108-11.

4. Pesch B, Harth V, Rabstein S, Baisch C, Schiffermann M, Pallapies D et al. Night work and breast cancer - results from the German GENICA study. Scandinavian journal of work, environment \& health. 2010;36(2):134-41.

5. Lie JA, Kjuus H, Zienolddiny S, Haugen A, Stevens RG, Kjaerheim K. Night work and breast cancer risk among Norwegian nurses: assessment by different exposure metrics. American journal of epidemiology. 2011;173(11):1272-9.

6. Hansen J, Lassen CF. Nested case-control study of night shift work and breast cancer risk among women in the Danish military. Occupational and environmental medicine. 2012;69(8):551-6.

7. Grundy A, Richardson H, Burstyn I, Lohrisch C, SenGupta SK, Lai AS et al. Increased risk of breast cancer associated with long-term shift work in Canada. Occupational and environmental medicine. 2013;70(12):831-8.

8. Menegaux F, Truong T, Anger A, Cordina-Duverger E, Lamkarkach F, Arveux P et al. Night work and breast cancer: a population-based case-control study in France (the CECILE study). International journal of cancer Journal international du cancer. 2013;132(4):924-31.

9. Fritschi L, Erren TC, Glass DC, Girschik J, Thomson AK, Saunders C et al. The association between different night shiftwork factors and breast cancer: a case-control study. British journal of cancer. 2013;109(9):2472-80.

10. Wang P, Ren FM, Lin Y, Su FX, Jia WH, Su XF et al. Night-shift work, sleep duration, daytime napping, and breast cancer risk. Sleep medicine. 2015;16(4):462-8.

11. Papantoniou K, Castano-Vinyals G, Espinosa A, Aragones N, Perez-Gomez B, Ardanaz E et al. Breast cancer risk and night shift work in a case-control study in a Spanish population. European journal of epidemiology. 2016;31(9):867-78.

12. Pronk A, Ji BT, Shu XO, Xue S, Yang G, Li HL et al. Night-shift work and breast cancer risk in a cohort of Chinese women. American journal of epidemiology. 2010;171(9):953-9.

13. Knutsson A, Alfredsson L, Karlsson B, Akerstedt T, Fransson EI, Westerholm P et al. Breast cancer among shift workers: results of the WOLF longitudinal cohort study. Scandinavian journal of work, environment \& health. 2013;39(2):170-7. 
14. Li W, Ray RM, Thomas DB, Davis S, Yost M, Breslow N et al. Shift work and breast cancer among women textile workers in Shanghai, China. Cancer causes \& control : CCC. 2015;26(1):143-50.

15. Koppes LL, Geuskens GA, Pronk A, Vermeulen RC, de Vroome EM. Night work and breast cancer risk in a general population prospective cohort study in The Netherlands. European journal of epidemiology. 2014;29(8):577-84.

16. Akerstedt T, Knutsson A, Narusyte J, Svedberg P, Kecklund G, Alexanderson K. Night work and breast cancer in women: a Swedish cohort study. BMJ open. 2015;5(4):e008127.

17. Travis RC, Balkwill A, Fensom GK, Appleby PN, Reeves GK, Wang XS et al. Night Shift Work and Breast Cancer Incidence: Three Prospective Studies and Meta-analysis of Published Studies. Journal of the National Cancer Institute. 2016;108(12).

18. Davis S, Mirick DK, Stevens RG. Night shift work, light at night, and risk of breast cancer. Journal of the National Cancer Institute. 2001;93(20):1557-62.

19. Jia Y, Lu Y, Wu K, Lin Q, Shen W, Zhu M et al. Does night work increase the risk of breast cancer? A systematic review and meta-analysis of epidemiological studies. Cancer epidemiology. 2013;37(3):197-206.

20. Ijaz S, Verbeek J, Seidler A, Lindbohm ML, Ojajarvi A, Orsini N et al. Night-shift work and breast cancer--a systematic review and meta-analysis. Scandinavian journal of work, environment \& health. 2013;39(5):431-47.

21. Kamdar BB, Tergas AI, Mateen FJ, Bhayani NH, Oh J. Night-shift work and risk of breast cancer: a systematic review and meta-analysis. Breast cancer research and treatment. 2013;138(1):291-301.

22. Wang F, Yeung KL, Chan WC, Kwok CC, Leung SL, Wu C et al. A meta-analysis on doseresponse relationship between night shift work and the risk of breast cancer. Annals of oncology : official journal of the European Society for Medical Oncology / ESMO. 2013;24(11):2724-32. 23. Stevens RG, Hansen J, Costa G, Haus E, Kauppinen T, Aronson KJ et al. Considerations of circadian impact for defining 'shift work' in cancer studies: IARC Working Group Report. Occupational and environmental medicine. 2011;68(2):154-62.

24. Higgins JP, Thompson SG, Deeks JJ, Altman DG. Measuring inconsistency in meta-analyses. BMJ. 2003;327(7414):557-60.

25. Hansen J, Stevens RG. Case-control study of shift-work and breast cancer risk in Danish nurses: impact of shift systems. European journal of cancer. 2012;48(11):1722-9.

26. Hansen J. Increased breast cancer risk among women who work predominantly at night. Epidemiology. 2001;12(1):74-7.

27. Schwartzbaum J, Ahlbom A, Feychting M. Cohort study of cancer risk among male and female shift workers. Scandinavian journal of work, environment \& health. 2007;33(5):336-43. 
28. Schernhammer ES, Laden F, Speizer FE, Willett WC, Hunter DJ, Kawachi I et al. Rotating night shifts and risk of breast cancer in women participating in the nurses' health study. Journal of the National Cancer Institute. 2001;93(20):1563-8.

29. Gu F, Han J, Laden F, Pan A, Caporaso NE, Stampfer MJ et al. Total and cause-specific mortality of U.S. nurses working rotating night shifts. American journal of preventive medicine. 2015;48(3):241-52.

30. Lie JA, Roessink J, Kjaerheim K. Breast cancer and night work among Norwegian nurses. Cancer causes \& control : CCC. 2006;17(1):39-44.

31. Tynes T, Hannevik M, Andersen A, Vistnes AI, Haldorsen T. Incidence of breast cancer in Norwegian female radio and telegraph operators. Cancer causes \& control : CCC. 1996;7(2):197204.

32. Wegrzyn LR, Tamimi RM, Rosner BA, Brown SB, Stevens RG, Eliassen AH et al. Rotating Night Shift Work and Risk of Breast Cancer in the Nurses' Health Studies. American journal of epidemiology. 2017.

33. Stevens RG. RE: Night Shift Work and Breast Cancer Incidence: Three Prospective Studies and Meta-analysis of Published Studies. Journal of the National Cancer Institute. 2017;109(4). 34. Straif K, Baan R, Grosse Y, Secretan B, Ghissassi FE, Bouvard V et al. Carcinogenicity of shiftwork, painting, and fire-fighting. Lancet Oncol. 2007;8(12):1065-6. 


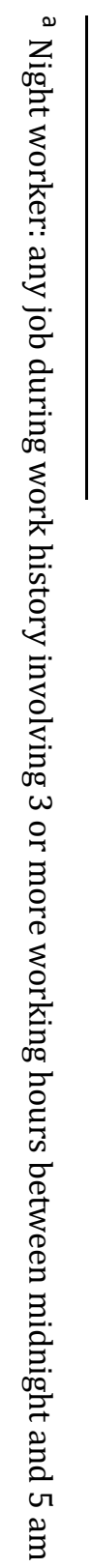

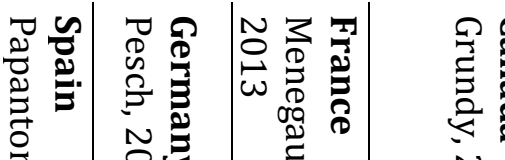

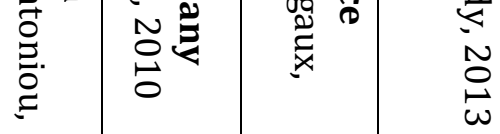

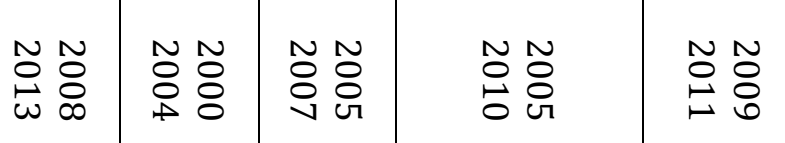

ֻ.

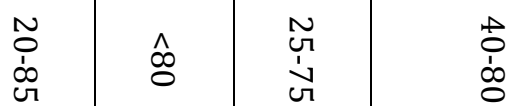

$b_{1}$
D
0

$\bullet$
$\infty$
$\infty$

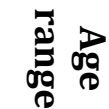

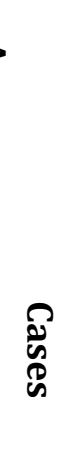

点

幽

它虽

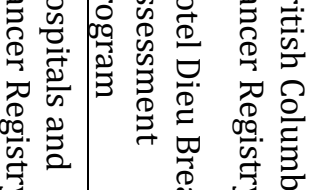

ڤே

$\stackrel{\overrightarrow{\bar{\sigma}}}{\mathrm{c}}$



离.

Э.

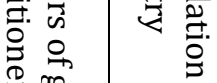

का

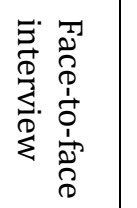

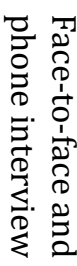

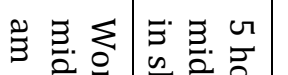

을

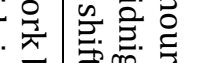

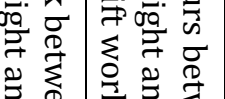

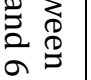

U

范

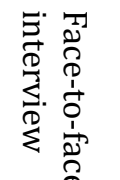

苞 过

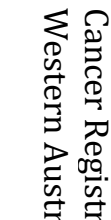

产. 这

ถุ

赵

$\frac{\overrightarrow{0}}{\frac{0}{2}}$

-

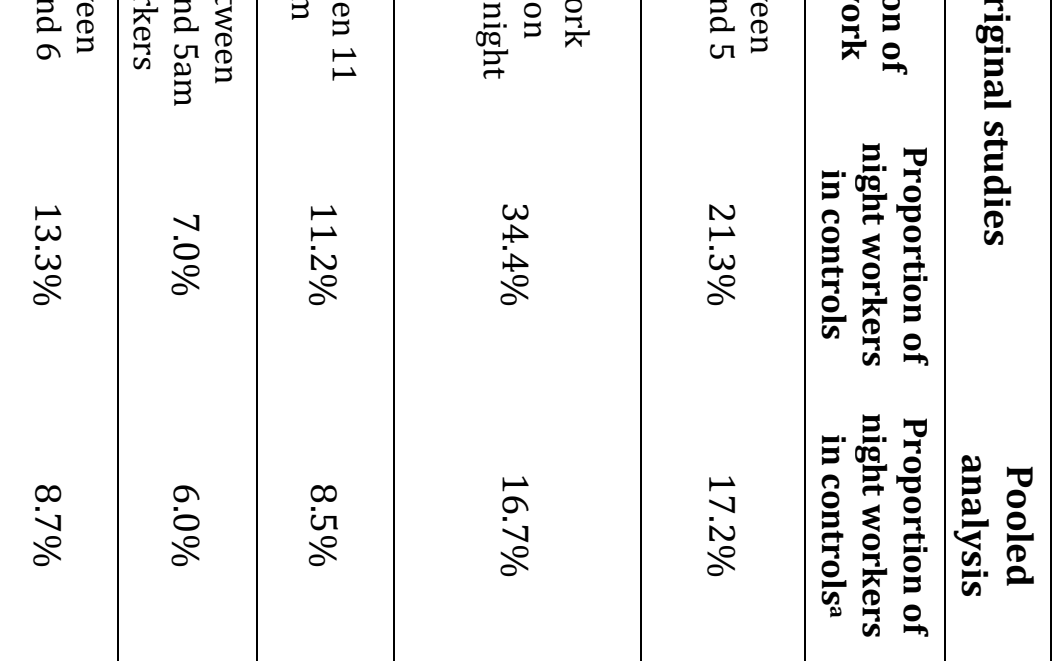




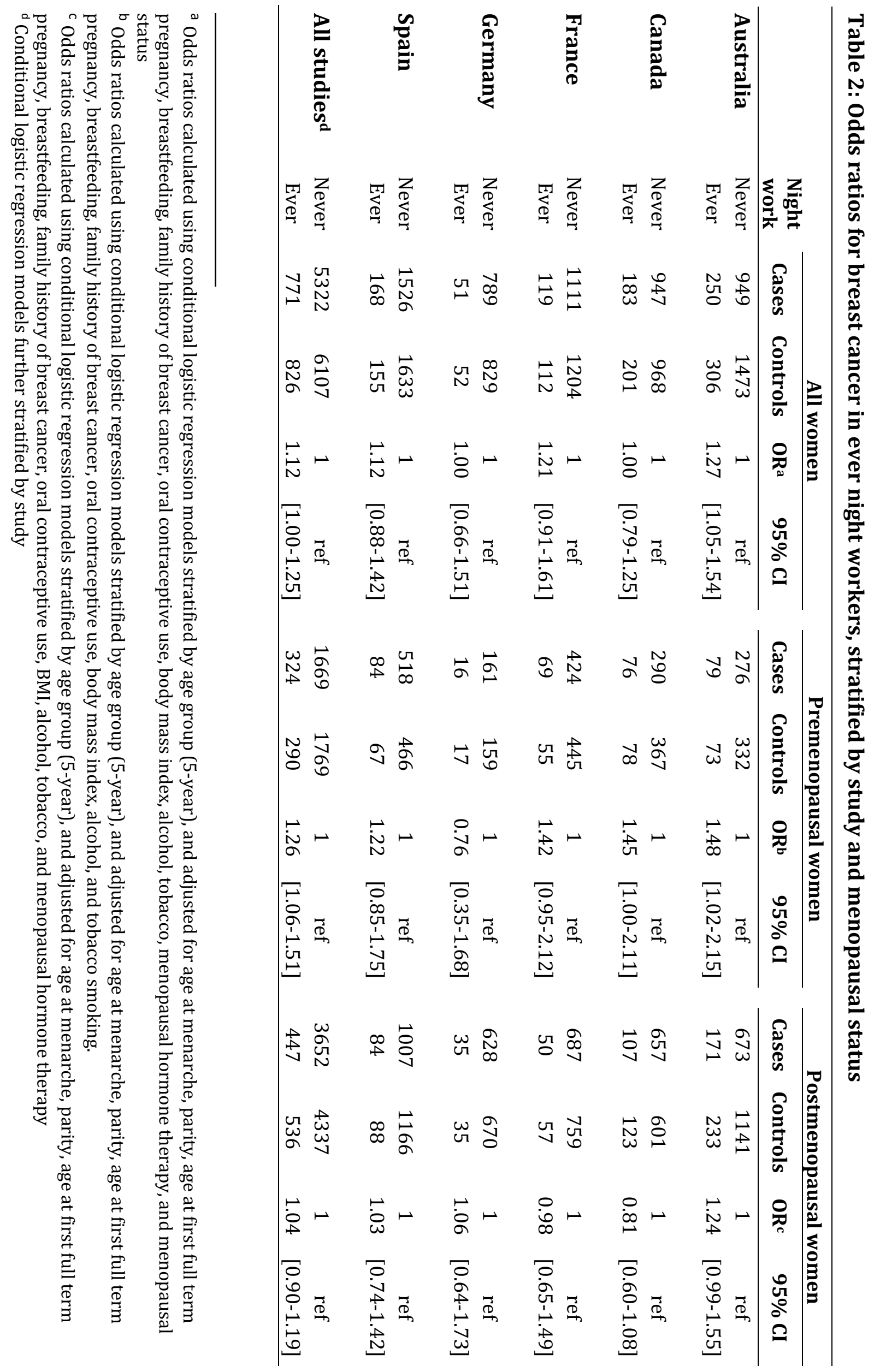




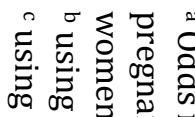

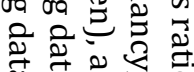
ए

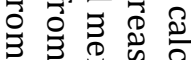

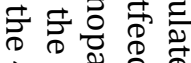
A U 己

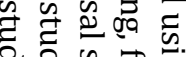
त. 织的远 류유 అ.

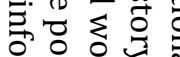

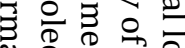
. . 은 . 崫 ริ 읓 을

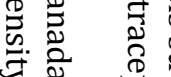

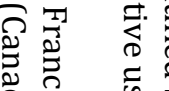
한

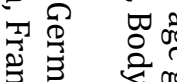

\%

की

言

क

造.

을

o

造

产

产



(1)

웅

要

옹

这

을

g.

흘

言

행

苋

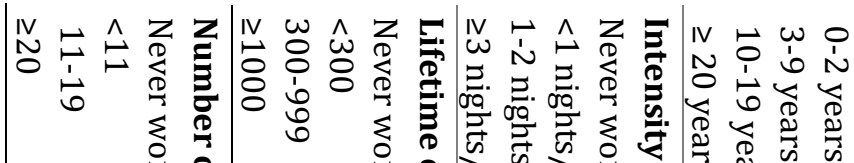

옻 욱

究

它.

कृ

\)

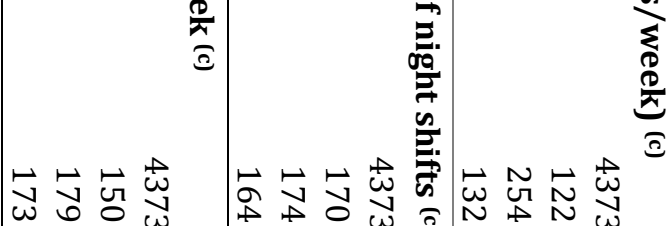

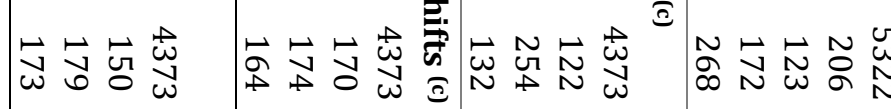

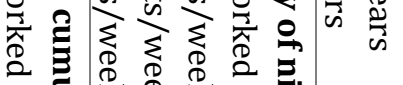

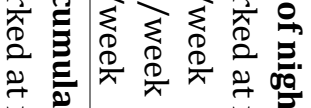

它.

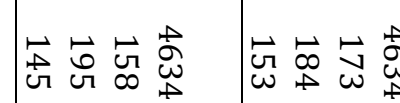

产客兽

Pदo

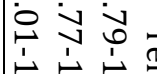

它富㹸

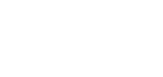

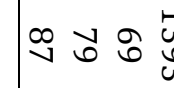

จ요 $\infty$

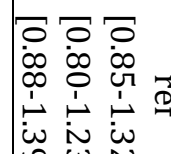

wِّ

促

灾灾芯

紫事

E.

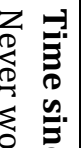

究

究

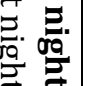

点

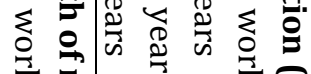

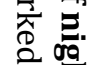

त्र

尝.

苛

$\Rightarrow$

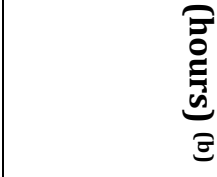

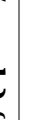

象

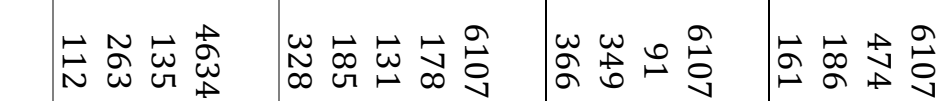

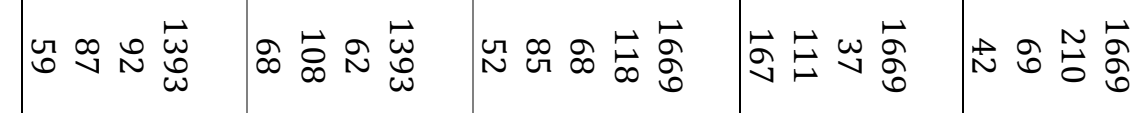

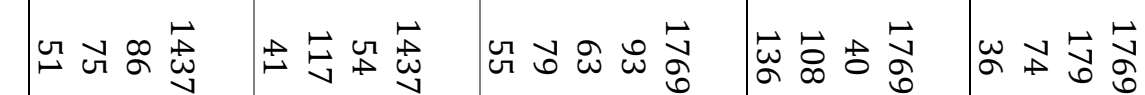

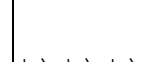

设萮荌

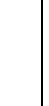

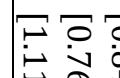

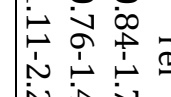

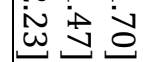

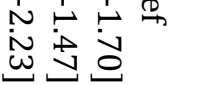

$\infty \stackrel{\infty}{\infty}$

㤐品总

₹客䯄

두웅

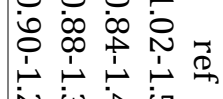

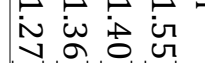

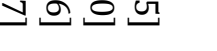

它完宫

岕空莣

$\overrightarrow{0} \dot{\infty}: \overrightarrow{\dot{\omega}}$

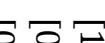




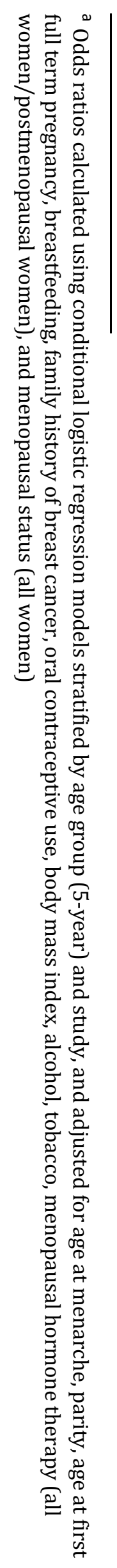

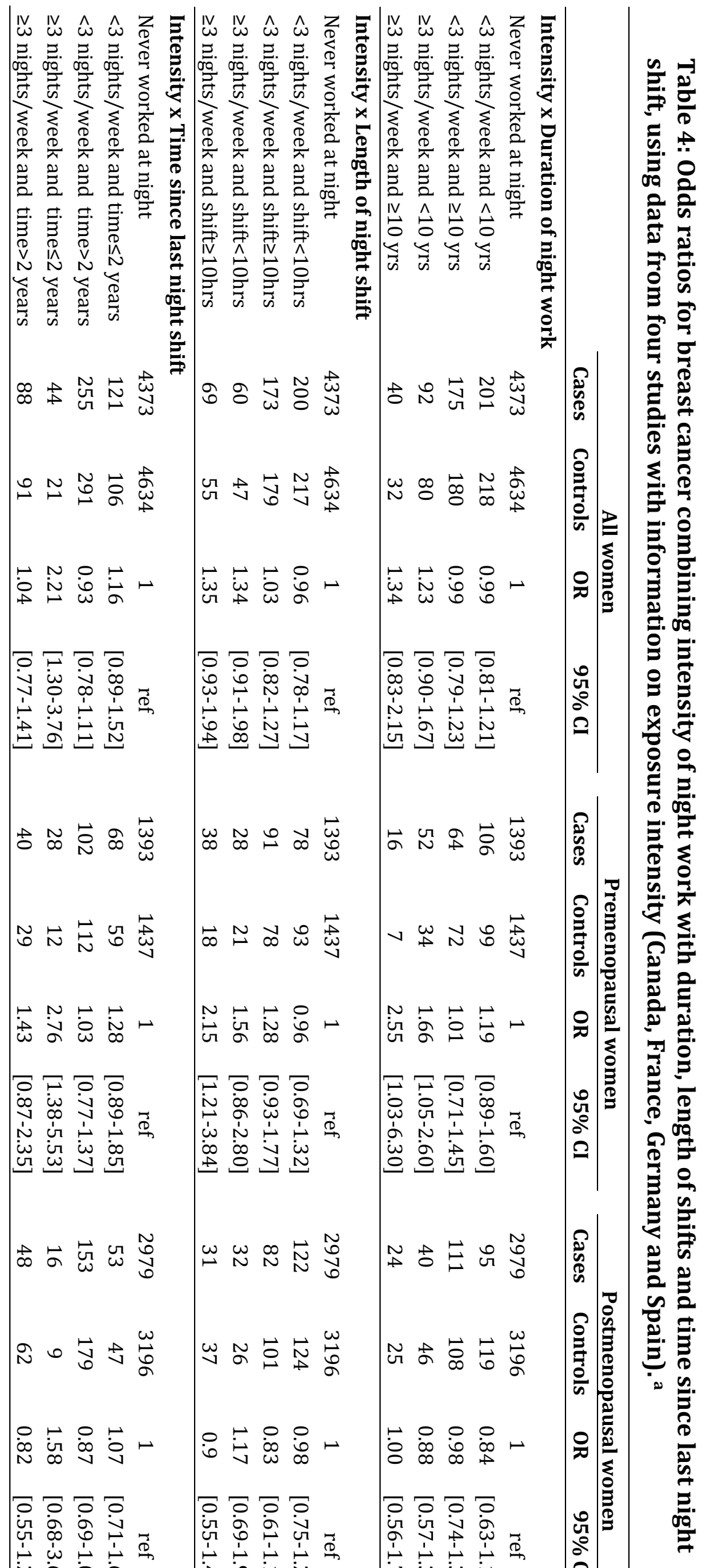




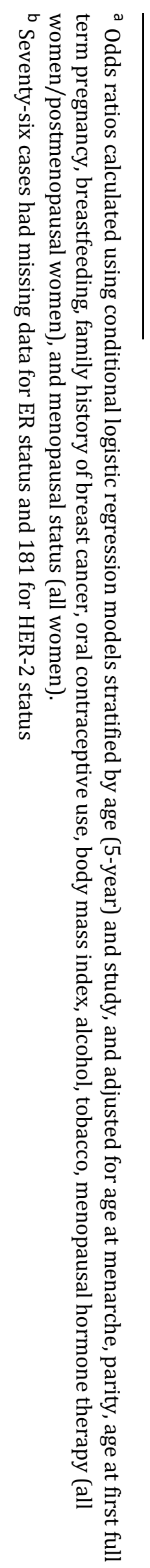

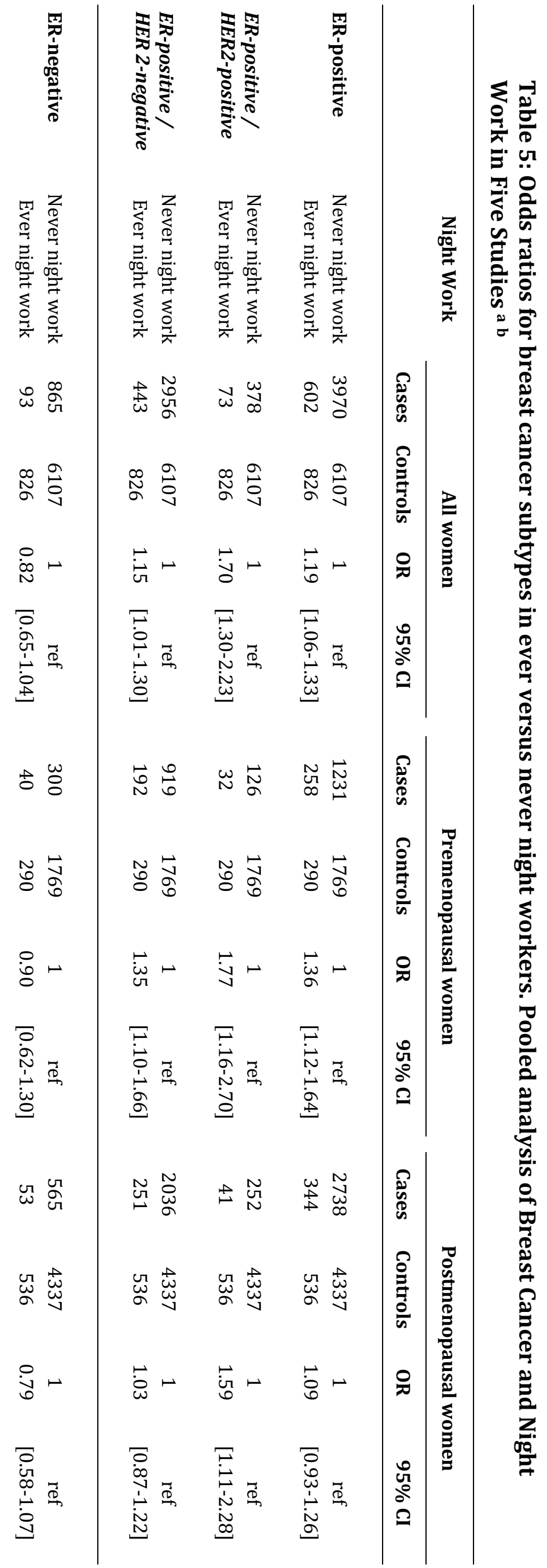

